\title{
Mineração
}

\section{Estudo de rotas de concentração para amostras de barita}

\section{(Investigation of concentration routes for barite samples)}

\author{
Rísia Magriotis Papini \\ Professora Adjunta Departamento de Engenharia de Minas da EEUFMG \\ E-mail: risia@demin.ufmg.br \\ Armando Corrêa de Araujo \\ Professor Adjunto Departamento de Engenharia de Minas da EEUFMG \\ E-mail:armando@demin.ufmg.br \\ Aline Pereira Leite \\ Aluna do Departamento de Engenharia de Minas da EEUFMG \\ E-mail:alinepl66@yahoo.com.br \\ Renata Corrêa da Silva \\ Aluna do Departamento de Engenharia de Minas da EEUFMG \\ E-mail:yullybr@yahoo.com.br
}

\section{Resumo}

No presente trabalho, foi estudada a possibilidade de flotação de 4 tipos de baritas, buscando-se mais de $60 \%$ de $\mathrm{BaO}$ e menos de $6 \%$ de $\mathrm{SiO}_{2}$ na recuperação.Procedeu-se a flotação, testando-se diferentes reagentes e combinações desses reagentes. A presença de sulfetos, nas amostras, levou, também, à busca de sua retirada na flotação, a fim de melhorar a recuperação e teor de $\mathrm{BaO}$. Nas quatro amostras de barita, tem-se que os melhores resultados obtidos demonstraram que o objetivo foi alcançado, ou seja, o teor de $\mathrm{BaO}$ ficou acima de $64 \%$, o teor de $\mathrm{SiO}_{2}$ abaixo de 3,3\% e a recuperação de $\mathrm{BaO}$ acima de 79,4\%. Para cada barita, porém, a rota de concentração foi diferente; na barita azul o cetil-estearil sulfato de sódio e o óleo de soja foram os melhores reagentes coletores, para a amostra de barita branca foi a mistura de sulfossucinato e ácido sulfônico que propiciou o melhor resultado, na barita T3 foi o óleo de soja e, na barita rejeito, o sulfossucinato.

Palavras-chave: Flotação, barita, coletores.

\begin{abstract}
In the present work flotation concentration of 4 types of barite was investigated, aiming for at least $60 \%$ of $\mathrm{BaO}$ and less than $6 \%$ of $\mathrm{SiO}_{2}$ in the concentrate. Flotation testing of different collectors, alone and in combinations, was performed. Because of the the presence of sulfides in the samples, a prior sulfide flotation step was performed for their removal in order to warrant the highest $\mathrm{BaO}$ final grades. The best results of the four barite samples showed that the objective was achieved with the $\mathrm{BaO}$ grade higher than $64 \%$, the $\mathrm{SiO}_{2}$ grade lower than 3,3\% at a $\mathrm{BaO}$ recovery of at least $79 \%$. The concentration route for each barite was different; in the blue barite the best collectors were cetylsterayl sulfate and soy bean oil, in the white barite was the mix of sulfossuccinate and sulfonic acid, in the T3 barite was the soy bean oil and in the so called tailing barite was the sulfossucinate.
\end{abstract}

Keywords: Flotation, barite, collectors. 


\section{Introdução}

A barita é um sulfato de bário $\left(\mathrm{BaSO}_{4}\right.$ ) composto de $65,7 \%$ de $\mathrm{BaO}$ e $34,3 \%$ de $\mathrm{SO}_{3}$ e sua densidade é em torno de $4,5 \mathrm{~g} / \mathrm{cm}^{3}$ e constitui-se no mais importante mineral de bário em termos de reservas, produção, utilização e obtenção do elemento bário. A elevada densidade da barita determina o seu uso em fluidos de perfuração de petróleo e gás e carga para diversos produtos. No Brasil, o consumo da barita tem a seguinte distribuição: indústria química 50\%, indústria petrolífera $35 \%$, outros (tinta, papéis, borracha, vidros, etc.) $15 \%$, todos com teor de $\mathrm{BaO}$ acima de $60 \%$.

O beneficiamento empregado para produção de concentrados de barita consiste de: britagem, lavagem em classificador espiral e moagem. A flotação é usada para recuperação da fração fina ou como processo de beneficiamento de minérios contendo fluorita ou sulfetos. A seletividade barita/quartzo é obtida utilizando-se ácidos carboxílicos, sulfato dodecil de sódio e sulfonatos.

As baritas estudadas são provenientes da baía de Camamu na Bahia. As características geológicas intrínsecas da região permitiram processos de mineralização, de reconhecido valor econômico. Nesse sentido, a região da baía de Camamu tem se imposto como um importante pólo mineiro, com extrações de manganês, gipsita, concentrações carbonosas, petróleo e barita. Destas, a barita destaca-se entre as mais importantes, devido a sua exploração nas ilhas centrais da baía, há mais de 60 anos.

\section{Materiais e métodos ${ }^{(5)}$}

As amostras trabalhadas são provenientes da Mineração Santa Rosa Pequeri/MG, coletadas na região de Camamu/BA. Abaixo se tem a denominação dessas amostras e seus pesos específicos.

- Barita $\mathrm{T} 3 \Rightarrow \mathrm{Pe}=3,87 \mathrm{~g} / \mathrm{cm}^{3}$

- Barita-rejeito $\Rightarrow \mathrm{Pe}=3,48 \mathrm{~g} / \mathrm{cm}^{3}$

- Barita branca $\Rightarrow \mathrm{Pe}=3,67 \mathrm{~g} / \mathrm{cm}^{3}$
- Barita azul $\Rightarrow \mathrm{Pe}=3,90 \mathrm{~g} / \mathrm{cm}^{3}$

Cada uma das amostras (Barita Azul, Rejeito, T3 e Branca) foram britadas separadamente de forma a obter $95 \%<10 \mathrm{~mm}$.

\subsection{Procedimento}

Para a realização dos ensaios de flotação, as amostras foram submetidas a moagem a úmido (20 minutos), obtendose mais de $95 \%<0,300 \mathrm{~mm}$. As massas abaixo de $0,300 \mathrm{~mm}$ e acima de $0,038 \mathrm{~mm}$ foram agrupadas e divididas em alíquotas de 500g, para a realização dos testes de flotação, e o material acima desta fração retornava na moagem. Apesar de não ser usual flotar material abaixo de $0,038 \mathrm{~mm}$, ao se verificar a sua representatividade na análise global (em torno de $20 \%$ ) e como se desejava a recuperação de material muito fino, alguns testes com essa granulometria foram realizados.

Na Tabela 1 está descrita a ordem de colocação dos reagentes, para as 4 baritas, assim como sua concentração nos testes de flotação para os melhores testes realizados. O tipo de reagente, a ordem de colocação e suas concentrações variaram para cada amostra de barita, onde levaram-se em conta, na escolha dos reagentes, a análise química e as difrações de raios X das amostras. Apenas as seguintes condições foram fixadas em todos os testes: a \% de sólidos $=70 \%$,o tempo de condicionamento espumantes $=1$ minuto e o tempo de condicionamento de coletores e ativador $=5$ minutos. As variáveis estudadas, para cada barita, separadamente, foram: tipo de coletor, mistura de coletores e concentração de coletor.

\subsection{Reagentes}

Foram utilzados, nesse trabalho, os principais reagentes, coletores de barita, encontrados no mercado, que permitem obter uma boa seletividade entre a barita e o quartzo. Esses coletores pertencem à família dos ácidos graxos, dos dodecil-sulfatos e dos sulfonatos.
Abaixo tem-se todos os reagentes empregados:

- Isopropil xantato de sódio (IPX) - coletor de sulfetos.

- Amil xantato de sódio (AMX) - coletor de sulfetos.

- Ácido sulfônico -AERO 825 (AERO) - coletor de barita.

- Sulfossucinato - KE 883 (KE) - coletor de barita.

- Cetil-estearil sulfato de sódio (CES) coletor de barita.

- Óleo de soja (OS) - coletor de barita.

- Sulfato de cobre (SC) - ativador de sulfeto.

- Metil isobutil carbinol (MIBC) - espumante.

- Óleo de pinho (OP) - espumante.

\subsection{Análise mineralógica e química}

As análises químicas das amostrascabeça, bem como dos testes, foram realizadas por fluorescência de raios $\mathrm{X}$ para os compostos $\mathrm{BaO} ; \mathrm{SO}_{3} ; \mathrm{SiO}_{2} ; \mathrm{Al}_{2} \mathrm{O}_{3}$; $\mathrm{Fe}_{2} \mathrm{O}_{3}$ e PF. Já as difrações de raios X das amostras-cabeça foram feitas através do método do pó total.

\subsection{Equipamentos utilizados}

- Célula de flotação Denver D12 com cubas de tamanhos variados.

- Britador de mandíbulas primário e secundário marca Arno.

- Moinho de bolhas com motor trifásico Weg.

- Moinho orbital tecnal modelo TE960.

\section{Resultados e discussão}

\subsection{Análise mineralógica e química}

Nas Tabelas 2 e 3, pode-se observar os resultados da análise química e da difração de raios $\mathrm{X}$ das amostras-cabeça das 4 baritas. 
Rísia Magriotis Papini et al.

Tabela 1 - Descrição dos melhores testes de flotação para as quatro amostras de barita.

\begin{tabular}{|c|c|c|c|c|c|c|c|c|c|}
\hline Teste & $\mathrm{pH}$ & Coletor 1 & $\begin{array}{l}\text { Conc. } \\
(g / t)\end{array}$ & Espum. & $\begin{array}{c}\text { Dosagem } \\
\text { (gotas) }\end{array}$ & $\begin{array}{c}\text { Coletor } \\
2,3,4\end{array}$ & $\begin{array}{l}\text { Conc. } \\
(g / t)\end{array}$ & Ativ. & $\begin{array}{c}\text { Conc. } \\
(g / t)\end{array}$ \\
\hline $1-A$ & 7,9 & OS & 1200 & - & - & CES & 300 & - & - \\
\hline $2-A$ & 7,5 & CES & 300 & - & - & OS & 1200 & - & - \\
\hline $3-B$ & 6,6 & IPX & 30 & MIBC & 1 & $\begin{array}{c}\mathrm{KE}+\mathrm{AERO} \\
\mathrm{OS}\end{array}$ & $\begin{array}{c}300+0,16 \mathrm{~g} \\
1200\end{array}$ & $\mathrm{SC}$ & 5000 \\
\hline $4^{*}-B$ & 6,9 & $\mathrm{KE}$ & 200 & - & & - & - & - & - \\
\hline $5-B$ & 6,5 & AMX & 30 & MIBC & 1 & $\begin{array}{l}\text { OS } \\
\text { CES }\end{array}$ & $\begin{array}{l}1200 \\
300\end{array}$ & - & - \\
\hline $6^{*}-\mathrm{B}$ & 6,6 & CES & 200 & - & - & - & - & - & - \\
\hline 7-T & 7,0 & IPX & 30 & MIBC & 1 & $\begin{array}{c}\text { KE } \\
\text { OS } \\
\text { AERO }\end{array}$ & $\begin{array}{c}300 \\
1200 \\
0,16 \mathrm{~g}\end{array}$ & $\mathrm{SC}$ & 5000 \\
\hline 8-T & 7,0 & IPX & 30 & MIBC & 1 & $\begin{array}{c}\text { OS } \\
\text { KE+AERO } \\
\text { AERO }\end{array}$ & $\begin{array}{c}1200 \\
300+0,16 \mathrm{~g} \\
0,2 \mathrm{~g}\end{array}$ & - & - \\
\hline $9^{*}-T$ & 7,6 & $\mathrm{KE}$ & 200 & - & - & - & - & - & - \\
\hline $10-\mathrm{T}$ & 7,2 & AMX & 30 & MIBC & 1 & $\begin{array}{l}\text { OS } \\
\text { CES }\end{array}$ & $\begin{array}{l}1200 \\
300\end{array}$ & - & - \\
\hline $11-\mathrm{T}$ & 7,2 & AMX & 30 & MIBC & 1 & $\begin{array}{l}\text { CES } \\
\text { OS }\end{array}$ & $\begin{array}{c}300 \\
1200\end{array}$ & - & - \\
\hline $12^{*}-\mathrm{T}$ & 7,2 & CES & 200 & - & - & - & - & - & - \\
\hline $13-\mathrm{R}$ & 5,0 & IPX & 50 & OP & 1 & $\mathrm{KE}$ & 200 & - & - \\
\hline $14-\mathrm{R}$ & 5,0 & IPX & 50 & MIBC & 1 & $\begin{array}{l}\mathrm{KE} \\
\mathrm{OS}\end{array}$ & $\begin{array}{c}200 \\
1200 \\
\end{array}$ & SC & 5000 \\
\hline $15^{*}-\mathrm{R}$ & 7,8 & $\mathrm{KE}$ & 600 & - & - & - & - & - & - \\
\hline $16-\mathrm{R}$ & 7,4 & AMX & 30 & MIBC & 1 & $\begin{array}{l}\text { OS } \\
\text { CES }\end{array}$ & $\begin{array}{l}1200 \\
300\end{array}$ & - & - \\
\hline $17-\mathrm{R}$ & 7,4 & AMX & 30 & MIBC & 1 & $\begin{array}{l}\text { CES } \\
\text { OS }\end{array}$ & $\begin{array}{c}300 \\
1200\end{array}$ & - & - \\
\hline $18^{*}-R$ & 7,4 & CES & 200 & - & - & - & - & - & - \\
\hline
\end{tabular}

Os testes são numerados em ordem e com a respectiva amostra de barita correspondente,

1-A(barita azul), 3-B(barita branca), 7-T(barita T3), 13-r(barita-rejeito).

Espum. = espumante, Conc. $=$ concentração, Ativ. $=$ ativador.

* Indica que a granulometria nesses testes foi de $-0,038 \mathrm{~mm}$. 
Estudo de rotas de concentração para amostras de barita

Tabela 2 - Análise química das 4 amostras-cabeça.

\begin{tabular}{c|c|c|c|c|c|c}
\hline AMOSTRA & $\% \mathrm{BaO}$ & $\% \mathrm{SO}_{3}$ & ${ }^{2} \mathrm{SiO}_{2}$ & ${ }^{2} \mathrm{Al}_{2} \mathbf{O}_{3}$ & $\% \mathrm{Fe}_{2} \mathbf{O}_{3}$ & PF \\
\hline Barita Azul & 59,8 & 32,3 & 7,97 & 0,95 & 0,16 & 0,39 \\
\hline Barita T3 & 57,6 & 28,9 & 10,6 & 1,28 & 0,63 & 1,03 \\
\hline Barita Branca & 57,7 & 29,1 & 10,4 & 1,65 & 0,46 & 0,67 \\
\hline Barita-Rejeito & 51,1 & 23,6 & 19,0 & 3,08 & 5,04 & 1,07 \\
\hline
\end{tabular}

Tabela 3 - Difração de raios X das amostras-cabeça de baritas.

\begin{tabular}{|c|c|c|c|c|c|c|}
\hline Amostra & Barita & Quartzo & Feldspato & Moscovita & Galena & Esfalerita \\
\hline Barita Azul & ++++ & ++ & ND & ND & ND & ND \\
\hline Barita Branca & ++++ & ++ & + & + & + & ND \\
\hline Branca A.M.* & ++++ & + & + & ND & ++ & ND \\
\hline Barita T3 & ++++ & ++ & + & ND & ND & ND \\
\hline T3 A.M.* & ++++ & ++ & + & + & ++ & ND \\
\hline Barita-Rejeito & +++ & +++ & ++ & + & ND & ND \\
\hline Rejeito A.M.* & +++ & +++ & + & ND & ND & ++ \\
\hline
\end{tabular}

${ }^{*}$ A.M. = Após Moagem.

Legenda: ++++: Alta concentração da fase. +++: Média concentração da fase.

++: Baixa concentração da fase. +: Traços da fase. ND: Não detectado.

Dos resultados de difração observados anteriormente, verifica-se que os principais constituintes das 4 amostras de barita são os minerais barita e quartzo. No caso da barita azul, são, praticamente, os únicos constituintes, mesmo depois da moagem. Na barita branca, temse a presença de galena. Observou-se que, após a moagem, a galena aparece na barita T3. No caso da barita-rejeito, a esfalerita também só é detectada após a moagem.

\subsection{Flotação}

Nas Tabelas 4 a 6, podem ser observados os balanços de massa dos testes de flotação para as 4 amostras de barita trabalhadas. Em todas as tabelas, as denominações FL 1,2,3 refere-se a flotado 1, 2, 3; AFUN é afundado; AL.REC. é alimentação recalculada.
Na Tabela 4, observa-se que o sulfato de cobre cumpriu sua função de ativar os sulfetos, porém a \% de massa obtida foi muito pequena.

Nota-se que o amil xantato não promove uma boa seletividade entre a barita e os sulfetos presentes no flotado, \% massa e teor de $\mathrm{BaO}$ muito altos (teste 5). Já o isopropil xantato fornece uma seletividade melhor e uma \% de massa bem pequena e teor de $\mathrm{BaO}$ mais baixo.

No teste 6, usando somente cetilestearil sulfato de sódio, observa-se uma recuperação de $\mathrm{BaO}$ maior no afundado (58\%) que no flotado (42\%), no teste 4 com KE883, observa-se que a recuperação no afundado também foi elevada (40\%), porém menor que no flotado (60\%). Já os teores de BaO, nos 2 afundados, foram muito elevados também
(48,2\% e 52,4\%, respectivamente). Dados referentes a outros testes de flotação e fotomicrografias da barita branca podem ser vistos na referência (6).

Na Tabela 5, em termos de flotação dos sulfetos, observa-se que o isopropil xantato permite a flotação somente dos sulfetos (galena e esfalerita, principalmente), com ou sem o uso do ativador sulfato de cobre, já o amil xantato flota muita barita junto com os sulfetos, fato comprovado pelos altos teores de $\mathrm{BaO}$ nos flotados 1 dos testes 10 e 11 e também de recuperação de $\mathrm{BaO}$.

Como visto para a barita branca, a flotação da barita T3 somente com cetilestearil (teste 12) ou somente com KE883 (teste 9) não fornece bons resultados, os afundados têm um teor de \%BaO alto e perdas elevadas de barita. 
Rísia Magriotis Papini et al.

Tabela 4 - Resultados dos testes de flotação para as baritas azul e branca.

\begin{tabular}{|c|c|c|c|c|c|c|c|c|c|}
\hline Teste & Produto & $\%$ Peso & $\% \mathrm{BaO}$ & $\% \mathrm{SO}_{3}$ & $\% \mathrm{SiO}_{2}$ & $\% \mathrm{Al}_{2} \mathrm{O}_{3}$ & $\% \mathrm{Fe}_{2} \mathrm{O}_{3}$ & PF & $\begin{array}{c}\text { \%Recup. } \\
\text { BaO }\end{array}$ \\
\hline \multirow{4}{*}{$1-A$} & FL1 & 83 & 62,3 & 31,0 & 3,56 & 0,27 & 0,17 & 0,32 & 97 \\
\hline & FL2 & 1 & 47,1 & 25,1 & 22,8 & 1,62 & 0,27 & 5,41 & 1 \\
\hline & AFUND & 16 & 6,43 & 3,14 & 85,9 & 1,63 & 0,33 & 0,60 & 2 \\
\hline & AL.REC. & 100 & 53,2 & 26,5 & 16,9 & 0,5 & 0,20 & 42,00 & 100 \\
\hline \multirow{4}{*}{$2-A$} & FL1 & 81 & 65,3 & 34,1 & 0,81 & 0,72 & 0,12 & 0,27 & 96 \\
\hline & FL2 & 7 & 20,7 & 10,3 & 57,3 & 2,31 & 0,54 & 2,91 & 3 \\
\hline & AFUND & 12 & 3,14 & 1,28 & 93,1 & 1,20 & 0,35 & 0,50 & 1 \\
\hline & AL.REC. & 100 & 54,7 & 39,5 & 15,8 & 0,89 & 0,30 & 0,48 & 100 \\
\hline \multirow{5}{*}{$3^{* *}-B$} & FL1 & 0,4 & 26,1 & 0,71 & 38,1 & 2,34 & 9,27 & * & 0,5 \\
\hline & FL2 & 94,3 & 55,4 & 29,1 & 11,1 & 1,13 & 0,26 & 3,90 & 94 \\
\hline & FL3 & 4,6 & 61,9 & 28,0 & 5,38 & 1,35 & 0,42 & 1,77 & 5 \\
\hline & AFUND & 0,7 & 28,2 & 14,6 & 45,6 & 4,75 & 0,67 & 0,82 & 0,5 \\
\hline & AL.REC. & 100 & 55,4 & 28,8 & 11,2 & 1,17 & 0,31 & 3,77 & 100 \\
\hline \multirow{3}{*}{ 4-B } & FL1 & 56 & 63,0 & 31,8 & 3,4 & 1,32 & 0,37 & 0,92 & 60 \\
\hline & AFUND & 44 & 52,4 & 26,3 & 18,5 & 2,72 & 0,94 & 1,11 & 40 \\
\hline & AL.REC. & 100 & 58,3 & 29,4 & 10 & 1,94 & 0,62 & 1,00 & 100 \\
\hline \multirow{5}{*}{$5-B$} & FL1 & 25,5 & 60,3 & 31,2 & 7,1 & 1,20 & 0,35 & 0,50 & 29,5 \\
\hline & FL2 & 57 & 62,6 & 31,0 & 5,01 & 1,08 & 0,28 & 0,26 & 69 \\
\hline & FL3 & 1,5 & 12,4 & 7,97 & 66 & 1,02 & 0,41 & 0,86 & 0,5 \\
\hline & AFUND & 16 & 2,28 & 0,86 & 87,8 & 2,78 & 0,66 & 1,31 & 1 \\
\hline & AL.REC. & 100 & 51,6 & 25,9 & 19,7 & 1,38 & 0,36 & 0,50 & 100 \\
\hline \multirow{3}{*}{$6-B$} & FL1 & 38 & 56,3 & 28,1 & 11 & 2,18 & 0,65 & 1,16 & 42 \\
\hline & AFUND & 62 & 48,2 & 23,6 & 22,9 & 1,81 & 0,42 & 0,71 & 58 \\
\hline & AL.REC. & 100 & 51,3 & 25,3 & 18,4 & 1,95 & 0,51 & 0,88 & 100 \\
\hline
\end{tabular}

*- massa insuficiente para dosagem.

$\left(^{* *}\right)$ - teores elevados de $\mathrm{ZnO} \sim 15 \%$ e $\mathrm{PbO} 6 \%$, além de cobre.

Observa-se, na Tabela 6, que há a mesma tendência em relação ao uso do isopropil xantato e amil xantato na flotação de sulfetos, ou seja, o amil xantato não fornece boa seletividade em relação a barita, com alto teor e recuperação de $\mathrm{BaO}$.
Ao contrário das baritas branca e T3, nota-se que a flotação somente com KE883 (flotando ou não os sulfetos-primeiramente) fornece bons resultados de teor e recuperação de $\mathrm{BaO}$ (testes 13,15).

A inversão na ordem de colocação dos reagentes (óleo de soja e cetil-este- aril) nos testes 16 e 17 não altera, significativamente, os resultados em termos de teor e recuperação de $\mathrm{BaO}$.

Nota-se que a seletividade na flotação das 4 amostras de barita, em relação ao quartzo $\left(\mathrm{SiO}_{2}\right)$ e aos outros possíveis minerais presentes, fornece me- 
Estudo de rotas de concentração para amostras de barita

Tabela 5 - Resultados dos testes de flotação para barita T3.

\begin{tabular}{|c|c|c|c|c|c|c|c|c|c|}
\hline Teste & Produto & \%Peso & $\% \mathrm{BaO}$ & $\% \mathrm{SO}_{3}$ & $\% \mathrm{SiO}_{2}$ & $\% \mathrm{Al}_{2} \mathrm{O}_{3}$ & $\% \mathrm{Fe}_{2} \mathrm{O}_{3}$ & PF & $\begin{array}{c}\begin{array}{c}\text { \%Recup. } \\
\text { BaO }\end{array} \\
\end{array}$ \\
\hline \multirow{6}{*}{ 7-T } & FL1 & 0 & $\star *$ & $\star \star$ & $* *$ & $* *$ & $\star *$ & $* *$ & - \\
\hline & FL2 & 1 & 52,4 & 23,8 & 15,4 & 1,35 & 1,09 & 1,74 & 1 \\
\hline & FL3 & 39 & 55,4 & 27,5 & 13,6 & 0,93 & 0,30 & 0,75 & 36 \\
\hline & FL4 & 59 & 64,7 & 32,6 & 1,71 & 0,76 & 0,15 & 0,21 & 62 \\
\hline & AFUND & 1 & 60,9 & 29,2 & 9,84 & 1,28 & 0,63 & 1 & 1 \\
\hline & AL.REC. & 100 & 60,8 & 30,3 & 6,0 & 0,88 & 0,22 & 0,44 & 100 \\
\hline \multirow{7}{*}{$8^{*}-T$} & FL1 & 0,7 & 3,06 & 26,8 & 13,5 & 0,65 & 4,05 & 6,56 & 0 \\
\hline & FL2 & 1 & 26,3 & 21,2 & 22,2 & 1,31 & 6,16 & 1,84 & 0,5 \\
\hline & FL3 & 19,3 & 64,6 & 34,1 & 1,12 & 0,87 & 0,17 & 0,25 & 22 \\
\hline & FL4 & 63 & 65,0 & 34,0 & 0,87 & 0,85 & 0,15 & 0,39 & 71 \\
\hline & FL5 & 10 & 33,5 & 17,2 & 42,9 & 1,61 & 0,35 & $<0,1$ & 5,5 \\
\hline & AFUND & 6 & 6,99 & 2,95 & 88,0 & 2,20 & 0,49 & 0,68 & 1 \\
\hline & AL.REC. & 100 & 57,5 & 17,2 & 10,7 & 1,01 & 0,28 & 0,41 & 100 \\
\hline \multirow{3}{*}{$9-T$} & FL1 & 58 & 63,1 & 32,6 & 2,51 & 1,12 & 0,30 & 0,63 & 61 \\
\hline & AFUND & 42 & 56,8 & 27,9 & 11,9 & 1,67 & 0,66 & 0,83 & 39 \\
\hline & AL.REC. & 100 & 60,5 & 30,6 & 6,43 & 1,35 & 0,45 & 0,71 & 100 \\
\hline \multirow{5}{*}{$10-\mathrm{T}$} & FL1 & 8 & 60,4 & 30,9 & 6,79 & 1,03 & 0,28 & 0,52 & 9,5 \\
\hline & FL2 & 74 & 63,9 & 32,3 & 1,80 & 0,74 & 0,24 & 0,35 & 89 \\
\hline & FL3 & 2 & 31,9 & 16,2 & 44,7 & 1,59 & 0,59 & 0,86 & 1 \\
\hline & AFUND & 16 & 1,28 & 0,38 & 94,9 & 1,84 & 0,35 & 0,33 & 0,5 \\
\hline & AL.REC. & 100 & 53,2 & 26,9 & 17,6 & 0,95 & 0,27 & 0,40 & 100 \\
\hline \multirow{5}{*}{$11-\mathrm{T}$} & FL1 & 30 & 62,1 & 31,4 & 2,67 & 0,9 & 0,24 & 0,30 & 50 \\
\hline & FL2 & 12 & 64,4 & 33,2 & 1,95 & 0,86 & 0,19 & 0,22 & 21 \\
\hline & FL3 & 51 & 18,6 & 19,6 & 56,9 & 1,66 & 0,71 & 1,87 & 26 \\
\hline & AFUND & 7 & 14,4 & 9,22 & 63,1 & 2,66 & 0,74 & 0,72 & 3 \\
\hline & AL.REC. & 100 & 36,9 & 24,1 & 34,5 & 1,40 & 0,51 & 1,13 & 100 \\
\hline \multirow{3}{*}{$12-\mathrm{T}$} & FL1 & 45 & 62,3 & 30,4 & 4,90 & 1,21 & 0,39 & 0,68 & 46 \\
\hline & AFUND & 55 & 59,5 & 29,9 & 8,11 & 1,49 & 0,48 & 0,71 & 54 \\
\hline & AL.REC. & 100 & 60,8 & 30,1 & 6,67 & 1,36 & 0,44 & 0,70 & 100 \\
\hline
\end{tabular}

* - amostra apresenta teores importantes de outros elementos como sódio, cobre, zinco e chumbo, além de magnésio, potássio e cálcio.

** - amostra não pode ser fundida devido ao alto teor de metais, principalmente chumbo. 
Rísia Magriotis Papini et al.

Tabela 6 - Resultados dos testes de flotação para barita-rejeito.

\begin{tabular}{|c|c|c|c|c|c|c|c|c|c|}
\hline Teste & Produto & $\%$ Peso & $\% \mathrm{BaO}$ & $\% \mathrm{SO}_{3}$ & $\% \mathrm{SiO}_{2}$ & $\% \mathrm{Al}_{2} \mathrm{O}_{3}$ & $\% \mathrm{Fe}_{2} \mathrm{O}_{3}$ & PF & $\begin{array}{c}\text { \%Recup. } \\
\text { BaO }\end{array}$ \\
\hline \multirow{4}{*}{$13-R$} & FL1 & 7 & 45,2 & 20,5 & 24,3 & 5,10 & 2,12 & 3,38 & 10 \\
\hline & FL2 & 47 & 58,9 & 30,0 & 9,65 & 1,94 & 0,68 & 0,75 & 88 \\
\hline & AFUND & 46 & 6,11 & 2,91 & 77,6 & 6,18 & 2,43 & 3,22 & 2 \\
\hline & AL.REC. & 100 & 31,6 & 16,9 & 6,89 & 4,1 & 1,58 & 2,07 & 100 \\
\hline \multirow{5}{*}{$14-\mathrm{R}$} & FL1 & 1 & 38,3 & 13,5 & 30,6 & 3,0 & 2,77 & 0,64 & 1 \\
\hline & FL2 & 9,5 & 60,4 & 30,6 & 7,77 & 1,31 & 0,42 & 0,60 & 13 \\
\hline & FL3 & 62,5 & 37,7 & 18,3 & 37,1 & 2,19 & 3,75 & 0,75 & 55 \\
\hline & AFUND & 27 & 50,4 & 24,4 & 20,7 & 2,36 & 2,41 & 0,83 & 31 \\
\hline & AL.REC. & 100 & 43,3 & 21,0 & 29,8 & 2,16 & 3,06 & 0,76 & 100 \\
\hline \multirow{3}{*}{$15-R$} & FL1 & 65 & 62,1 & 31,2 & 3,26 & 1,25 & 0,39 & 2,91 & 92 \\
\hline & AFUND & 35 & 9,74 & 6,7 & 60,9 & 7,87 & 3,91 & ** & 8 \\
\hline & AL.REC. & 100 & 43,8 & 22,6 & 23,4 & 3,57 & 1,62 & 1,89 & 100 \\
\hline \multirow{5}{*}{$16-R$} & FL1 & 39 & 56,8 & 29,2 & 12,1 & 1,41 & 0,71 & 0,45 & 49 \\
\hline & FL2 & 38 & 58,5 & 28,4 & 9,83 & 1,35 & 1,05 & 0,62 & 50 \\
\hline & FL3 & 1 & 7,43 & 3,42 & 80,6 & 3,71 & 0,88 & 3,70 & 0,5 \\
\hline & AFUND & 22 & 1,01 & 0,13 & 88,2 & 5,49 & 0,87 & 0,61 & 0,5 \\
\hline & AL.REC. & 100 & 44,5 & 22,2 & 28,9 & 2,32 & 0,88 & 0,58 & 100 \\
\hline \multirow{5}{*}{$17-\mathrm{R}$} & FL1 & 36 & 58,9 & 30,4 & 8,85 & 1,26 & 0,73 & 0,37 & 50 \\
\hline & FL2 & 28 & 62,1 & 31,8 & 5,35 & 1,12 & 0,33 & 0,32 & 40 \\
\hline & FL3 & 16 & 26,7 & 13,9 & 45,4 & 3,34 & 1,68 & 1,89 & 9 \\
\hline & AFUND & 20 & 1,48 & 0,48 & 87,9 & 5,39 & 0,66 & 0,8 & 1 \\
\hline & AL.REC. & 100 & 43,3 & 22,1 & 20,5 & 2,37 & 0,76 & 0,68 & 100 \\
\hline \multirow{3}{*}{$18-R$} & FL1 & 63 & 56,0 & 28,3 & 10,6 & 2,47 & 1,18 & 1,36 & 68 \\
\hline & AFUND & 37 & 45,3 & 22,2 & 23,2 & 4,5 & 2,37 & 1,25 & 32 \\
\hline & AL.REC. & 100 & 52,0 & 26,0 & 15,3 & 3,22 & 1,62 & 1,32 & 100 \\
\hline
\end{tabular}

** - massa insuficiente para dosagem.

lhores resultados sempre no primeiro flotado de barita, após a retirada dos sulfetos, quando presentes, no segundo e terceiro flotados essa seletividade diminui muito.

\section{Conclusões}

- Na barita azul os reagentes cetil-estearil e o óleo de soja são bons coletores, elevando o teor de $\mathrm{BaO}$ para $65 \%$, o de $\mathrm{SiO}_{2}$ abaixando para $0,8 \%$ com uma recuperação média de $99 \%$ de $\mathrm{BaO}$.
- Na barita branca, T3 e rejeito, o isopropil xantato oferece uma boa seletividade na flotação de sulfetos.

- Na barita branca, os melhores resultados na flotação de barita, em relação aos testes executados, foram 
obtidos com a mistura de KE883 e aero825 $\left(\% \mathrm{BaO}=63 \%, \% \mathrm{SiO}_{2}=3 \%\right)$ e recuperação $\mathrm{BaO}=88,4 \%$.

- Na barita T3, em termos de teor de $\mathrm{BaO}$, todos os reagentes testados fornecem bons resultados (acima 63\%); já, em termos de recuperação, o melhor resultado(85\%) foi obtido com o óleo de soja.

- Na barita-rejeito, em termos de teor de $\mathrm{BaO}$, todos os reagentes testados também fornecem bons resultados (acima de 62\%), já, em termos de recuperação, os melhores resultados foram obtidos com o uso de KE883 (acima de 79\%);

- Para as 4 amostras de barita trabalhadas, os melhores resultados, em termos de teor e recuperação, foram:

- Barita azul: BaO de 59,9 para 65,3\%, $\mathrm{SiO}_{2}$ de 7,9 para $0,8 \%$, recuperação de $99 \%$.

- Barita-branca: BaO de 57 para 63\%, $\mathrm{SiO}_{2}$ de 10,6 para 3\%, recuperação de $88,4 \%$.

- Barita T3: BaO de 57 para 65\%, $\mathrm{SiO}_{2}$ de 10,6 para $1,2 \%$, recuperação de $85,4 \%$.
- Barita-rejeito: $\mathrm{BaO}$ de 51 para 64\%, $\mathrm{SiO}_{2}$ de 19,2 para 3,5\%, recuperação de $79,4 \%$.

- O principal mineral contaminante, quartzo, foi removido com bastante sucesso em todas as amostras. Deve-se ressaltar que os ensaios realizados ainda não contemplaram etapas de limpeza e relimpeza (cleaner/recleaner), o que, certamente, irá reduzir o teor do contaminante quartzo em níveis ainda mais baixos dos que já foram obtidos.

\section{Agradecimentos}

A Mineração Santa Rosa, Pequeri-MG, pelo fornecimento das amostras e liberação do estudo.

\section{Referências bibliográficas}

1. KLEIN, C., HURLBURT, C. Jr. Manual of Mineralogy. 20 ed., Editora Wiley, 1984. p.425-427.

2. GUIMARÃES, R. C. Aspectos relevantes da separação de barita em minério fosfático através de flotação. CPGEM, 1997. 100p. (Tese de doutorado).

3. LIMA, W. S., MANSO, C. L. C., ANDRADE, E. J., GRILLO, J.L. Bacias sedimentares brasileiras. IGC-UnB, 1984. 12p.

4. LUZ, A. B., LINS, F. F. Barita. In: Rochas e minerais industriais. Rio de Janeiro: CETEM/ MCT, 2005. cap.12, p.263-277. 726p.

5. PAPINI, R.M., ARAÚJO, A.C. et al. Projeto Barita - Mineração Santa Rosa. FCO, 2005. 74p. (Relatório Técnico).

6. PAPINI, R. M., ARAUJO, A. C., LEITE, A. P., SILVA, R. C. Characterization and definition of parameters to concentration route of white barite by flotation. In: JORNADAS ARGENTINAS DE TRATAMIENTO DE MINERALES, 8. Actas... San Juan: Ed. UNSJ, 2006. p.21-30.

Artigo recebido em 28/09/2006 e aprovado em 03/05/2007.

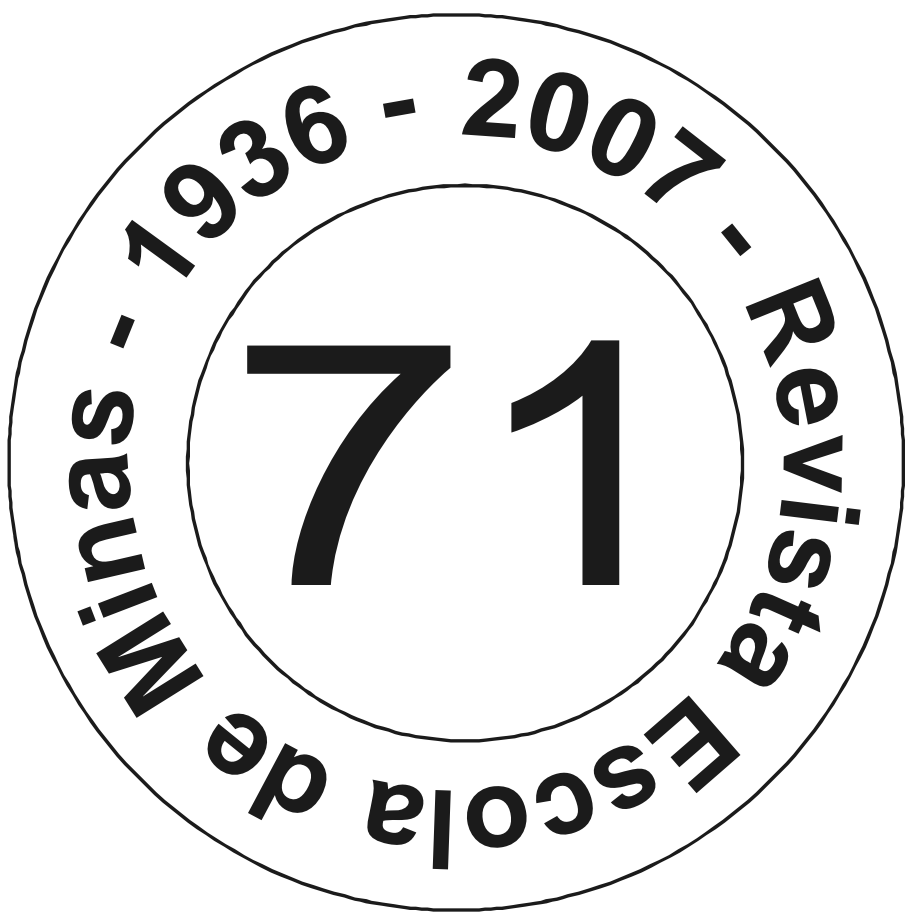

